\title{
Chronisch-entzündliche Darmerkrankungen Vernetzt und informiert den Alltag meistern
}

— Da Chronisch-entzündliche Darmerkrankungen (CED) sich erstmals meist in der Jugend oder im jungen Erwachsenenalter manifestieren und die gesamte Berufszeit andauern können, sind Therapie und Betreuung aufwendig. "Chronisch-entzündliche Darmerkrankungen haben auf alle Lebensbereiche der meist jungen Patienten erhebliche Auswirkungen, Probleme in Bereichen wie Ausbildung, Berufswahl, Partnerschaften oder Sport können mit ArztPatienten-Kontakten allein nicht umfassend bearbeitet werden", sagte Dr. med. Lars Fechner bei einer Veranstaltung des Unternehmens Takeda in Köln. Fechner ist niedergelassener Gastroenterologe in Halle an der Saale mit Tätigkeitsschwerpunkt CED. Diese meist junge Patientengeneration habe ein hohes Bedürfnis, sich über die Krankheit zu informieren und über die Frage, wie der Lebensstil optimal angepasst werden kann - die meisten nutzten dazu die neuen Medien. Eine longitudinale vergleichende Da- tenbankanalyse habe ergeben, dass Patientenunterstützungsprogramme die Zufriedenheit von CED-Patienten erhöhen und die krankheitsspezifischen Kosten senken, berichtete Fechner. Einzigartig für das Supportprogramm "myvyo TM" sei, dass zusätzlich zu einer interaktiven OnlinePlattform (www.CED-trotzdem-ich.de) auf Wunsch die Möglichkeit bestehe, sich individuell von speziell ausgebildeten Coachs beraten zu lassen: im telefonischen Gespräch oder per Chat. "myvyo TM“ sei aus rechtlichen Gründen nur Patienten zugänglich, die Vedolizumab (Entyvio ${ }^{\oplus}$ ) erhalten, erklärte Ulrike Talkenberg, Patient Care Manager CED bei Takeda.

„Der CED-Patient zwischen Informationsbedürfnis, Therapieoptionen und Alltag - welche Unterstützung können moderne Wege in der Patientenkommunikation bieten?"; Köln, 20. Januar 2017 (Veranstalter: Takeda Pharma Vertrieb $\mathrm{GmbH} \&$ Co. KG)

\section{Broschüre \\ Ernährung bei Hautkrebs}

— Die Broschüre „Ernährung bei Hautkrebs" haben Experten aus Medizin, Pflege und Patientenversorgung im Rahmen eines Workshops erarbeitet. Sie gibt praktische Tipps und beantwortet ganz konkrete Fragen von Patienten mit weißem und schwarzem Hautkrebs. Dies kann das Gespräch mit dem Patienten unterstützen. Die Broschüre kann im Internet bestellt oder als PDF herunter geladen werden. Auch eine Bestellung per Post oder Telefon ist möglich: Roche Pharma AG, Emil-BarellStraße 1, 79639 Grenzach-Wyhlen; Tel.: 07624/14-2014.

www.info-hautkrebs.de

\section{Modellierbarer Hautschutzring Zweifacher Schutz, einfache Anwendung}

— Ganz gleich, ob kleine oder Stomata mit großem Durchmesser abzudichten sind, ob eher leichte oder starke Unebenheiten auszugleichen sind, um Leckagen zu ver-

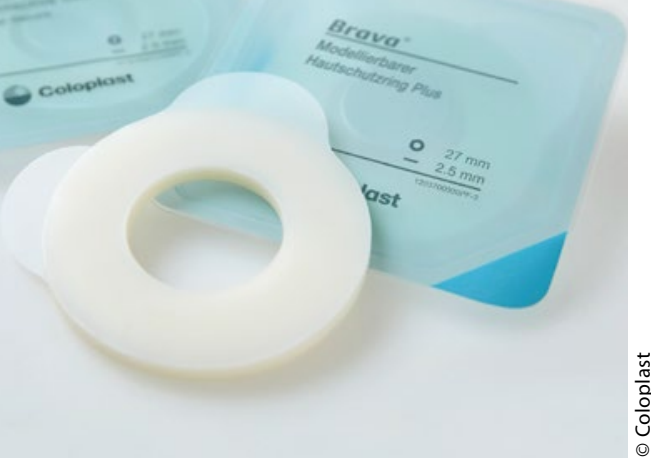

meiden, oder ob irritierte Haut geschützt werden soll: Mit dem Brava ${ }^{\circledR}$ Modellierbaren Hautschutzring Plus gibt es jetzt ein ganzes Ringsortiment, um so für jedes Stoma einen passenden Ring anbieten zu können. Dank spezieller Rezeptur bietet er die ideale Kombination aus Absorptionsvermögen und Beständigkeit. Durch die BodyFit ${ }^{\oplus}$ Technologie hat der neue Hautschutzring eine hohe Anpassungsfähigkeit, die für eine zuverlässige Abdichtung sorgt. Dank einer neuen Polymerformel ist er erosionsbeständig und löst sich auch nach lang anhaltendem Kontakt mit aggressiven Ausscheidungen nicht auf. Denn aufgrund der speziellen Rezeptur kann der Ring hohe Mengen an Feuchtigkeit absorbieren. Er hat eine sehr hautfreundliche Zusammensetzung, so dass er auch auf feuchter oder gereizter Haut eingesetzt werden kann.

Das Besondere: Der Brava ${ }^{\circledR}$ Modellierbarer Hautschutzring Plus lässt sich unkompliziert in die gewünschte Form und Größe modellieren. Er behält die Form auch während des Tragens - selbst bei starker Bewegung. Der Ring ist extrem vielfältig in der Anwendung: Der Stomaträger kann ihn bei Bedarf zu einer Rolle formen oder durchschneiden. Er lässt sich stapeln, etwa um Konvexitäten zu verstärken, oder schön ausformen, um Vertiefungen auszukleiden. Und noch ein großes Plus: Der Hautschutzring löst sich zusammen mit der Stomaplatte $a b$, wobei sich eventuelle Rückstände einfach entfernen lassen.

Der Brava ${ }^{\circledast}$ Modellierbare Hautschutzring Plus ist in zwei Stärken ( 2,5 und 4,2 mm) und drei verschiedenen Starterlochgrößen (18, 27 und $34 \mathrm{~mm}$ ) erhältlich. Dies ermöglicht eine einfache Anwendung und sichere Abdichtung auch bei größeren Stomadurchmessern, doppelläufigen Stomata und unterschiedlichsten Stomaumgebungen.

www.bravaplus.coloplast.de 